\title{
Phase Based Level Set Segmentation of Ultrasound Images
}

\author{
A. Belaid, D. Boukerroui, Y. Maingourd and J-F. Lerallut, Senior Member, IEEE
}

\begin{abstract}
Ultrasound images segmentation is a difficult problem due to speckle noise, low contrast and local changes of intensity. Intensity based methods do not perform particularly well on ultrasound images. However, it has been previously shown that these images respond well to local phase-based methods which are theoretically intensity-invariant. Here, we use level set propagation to capture the left ventricle boundaries. This uses a new speed term based on local phase and local orientation derived from the monogenic signal, which makes the algorithm robust to attenuation artefact. Furthermore, we use Cauchy kernels, instead of the commonly used log-Gabor, as pair of quadrature filters for the feature extraction. Preliminary results show that the proposed method can robustly handle noise, and captures well the low contrast boundaries.
\end{abstract}

Index Terms-Echocardiography, Segmentation, Local phase, Monogenic signal, Level set.

\section{INTRODUCTION}

$\mathbf{U}$ LTRASOUND imaging has many advantages: it is noninvasive, provides images in real time and requires a relatively lightweight material. However, these images are known to have low signal-to-noise ratio, low contrast, and high amounts of speckle. These characteristics make segmentation difficult and therefore complicate the diagnosis task.

A correct segmentation of structures is crucial in many medical applications, e.g. diagnosis, surgical planning, simulation and training. The most popular approach has been to treat echocardiographic endocardial segmentation as a contour finding approach. This is not straightforward as the contrast around the left ventricle chamber boundaries varies depending on its relative orientation to the transducer direction, and to attenuation. Thus conventional intensity gradient-based methods have had limited success on typical clinical images [1]. To avoid this drawback, phase based approach offers a good alternative, it makes the approach robust to attenuation artifacts.

This paper concerns the development of a novel segmentation method of the left ventricle within the level set framework. This uses local phase information derived from the monogenic signal, which is a multidimensional extension of the analytic signal [2], [3], [4]. Phase information has

Manuscript received July 09, 2009. This work was supported by a grant of the Regional Council of Picardie.

A. Belaid, D. Boukerroui and J. F. Lerallut are with Université de Technologie de Compiègne, CNRS, Heudiasyc UMR 6599 Centre de Recherche de Royallieu, BP 20529 - 60205 Compiègne Cedex, France ( e-mail:\{ahror.belaid, djamal.boukerroui, jean-francois.lerallut\}@ hds.utc.fr).

Y. Maingourd is the head of the Pediatric Échocardiographic Center, CHU Amiens, France (e-mail: maingourd.yves@chu-amiens.fr). been used in numerous applications in the literature, such as image registration [5], stereo matching [6], filtering [7], enhancement [8], corner and edges detection [9], [10] and segmentation [11], [12], [13], [14]. Phase-based processing has attracted a lot of attention in image analysis, but probably not still enough in ultrasound image segmentation, see [1]. Our idea is to use a novel speed function, which combines the local phase and local orientation in order to detect boundaries in low contrast regions.

In the next section, we describe the extraction of local properties (phase and amplitude) from 1D and 2D signals. Section 3 presents our proposed segmentation method. Finally, we conclude the paper with preliminary results on echocardiographic images.

\section{BACKGROUND}

Openheimer and Lim [15] showed that constructing two synthetic images, one of them containing the phase and the other containing the amplitude, only the image containing the phase is seen, although deteriorated. The information carried by phase of the picture appears much more significant than information carried by amplitude. Indeed, the phase informs us about the location and orientation of image boundaries, while the module provides only information on their intensity.

\section{A. The Monogenic signal}

To extract the local properties (Amplitude and Phase) of a 1D signal $f(x)$ we need to represent it in its analytic form [16], [17]:

$$
f_{A}(x)=f(x)-i f_{\mathcal{H}}(x),
$$

where $i=\sqrt{-1}$ and $f_{\mathcal{H}}(x)$ is the Hilbert transform of $f(x)$. The local amplitude (energy) and local phase of $f(x)$ are given by

$$
\begin{aligned}
A(x)=\left\|f_{A}(x)\right\| & =\sqrt{f^{2}(x)+f_{\mathcal{H}}^{2}(x)}, \\
\phi(x) & =\arctan \left(f_{\mathcal{H}}(x) / f(x)\right) .
\end{aligned}
$$

Recently, Felsberg and Sommer [3], [4], proposed a novel two-dimensional generalization of the analytic signal based on the Riesz transform, which is used instead of the Hilbert transform. Also, they proposed a 2D isotropic analytic signal, called monogenic signal. This representation preserves the core properties of the 1D analytic signal that decomposes a signal into information about structure (local phase) and energy (local amplitude). Several applications of the monogenic signal have been already realized (see eg. [17], [18]). 
Felsberg and Sommer introduced the following filters in the frequency domain:

$$
H_{1}\left(u_{1}, u_{2}\right)=i \frac{u_{1}}{\sqrt{u_{1}^{2}+u_{2}^{2}}}, \quad H_{2}\left(u_{1}, u_{2}\right)=i \frac{u_{2}}{\sqrt{u_{1}^{2}+u_{2}^{2}}} .
$$

The spatial representation of the above filters is given by:

$$
h_{1}\left(x_{1}, x_{2}\right)=\frac{-x_{1}}{2 \pi\left(x_{1}^{2}+x_{2}^{2}\right)^{\frac{3}{2}}}, \quad h_{2}\left(x_{1}, x_{2}\right)=\frac{-x_{2}}{2 \pi\left(x_{1}^{2}+x_{2}^{2}\right)^{\frac{3}{2}}} .
$$

The monogenic signal $\mathbf{f}_{M}$ is then defined as a $3 \mathrm{D}$ vector formed by the signal $f\left(x_{1}, x_{2}\right)$ with its Riesz transform $\mathbf{f}_{\mathcal{R}}=$ $(\mathbf{h} * f)\left(x_{1}, x_{2}\right)$, with $\mathbf{h}=\left(h_{1}, h_{2}\right)$

$$
\mathbf{f}_{M}\left(x_{1}, x_{2}\right)=(f, \mathbf{h} * f)\left(x_{1}, x_{2}\right)=\left(f, \mathbf{f}_{\mathcal{R}}\right)\left(x_{1}, x_{2}\right) .
$$

In the $\mathrm{nD}$ case, the local phase is associated to a given local orientation due to the fact that structural information is related to a given orientation.

The concept of Local phase-vector is proposed by Felsberg in [2], [4] and is defined by:

$$
\begin{aligned}
\boldsymbol{\varphi} & =\phi \cdot \mathbf{r}, \\
\boldsymbol{\varphi} & =\arctan \left(\frac{\left\|\mathbf{f}_{\mathcal{R}}\right\|}{f}\right) \frac{\mathbf{f}_{\mathcal{R}}}{\left\|\mathbf{f}_{\mathcal{R}}\right\|},
\end{aligned}
$$

where $\arctan (\cdot) \in[0, \pi]$. The monogenic phase $\varphi$ (local phase-vector), is similar to the product of $1 \mathrm{D}$ local phase multiplied by the orientation vector, if the underlying signal is $i 1 \mathrm{D}$ (intrinsically one dimensional signals). The defined local phase vector can be interpreted as a rotation vector, which magnitude corresponds to the phase angle between the real signal and the monogenic signal, (see Fig. 1). In contrast to the $1 \mathrm{D}$ case, the phase now includes additional geometric information. The monogenic phase completely characterizes the local gray-level transition of an image (i.e. local structure) as long as the image is locally $i 1 \mathrm{D}$, given that the phase has been defined with respect to a given orientation [19], (see Fig.2). The phase vector orientation $\mathbf{r}$ represents the local orientation of the image and is defined by the following relation:

$$
\theta=\arctan \left(\frac{h_{2} * f}{h_{1} * f}\right),
$$

where $\arctan (\cdot) \in[0, \pi]$.

\section{B. Quadrature filters}

In practical applications, the local properties are estimated using a pair of bandpass quadrature filters. The monogenic signal is often constructed as

$$
\begin{aligned}
\mathbf{f}_{M}\left(x_{1}, x_{2} ; s\right) & =(c * f, c * \mathbf{h} * f)\left(x_{1}, x_{2}\right) \\
& =\left(c * f, c * \mathbf{f}_{\mathcal{R}}\right)\left(x_{1}, x_{2}\right),
\end{aligned}
$$

where $c\left(x_{1}, x_{2} ; s\right)$ is the spatial domain representation of the isotropic bandpass filter and $s$ is a scaling parameter. So it can be represented by scalar valued even and vector valued odd filtered responses, with the following simple tick:

$$
\begin{aligned}
\text { even } & =c * f, \\
\text { odd } & =\left(c * h_{1} * f, c * h_{2} * f\right) .
\end{aligned}
$$

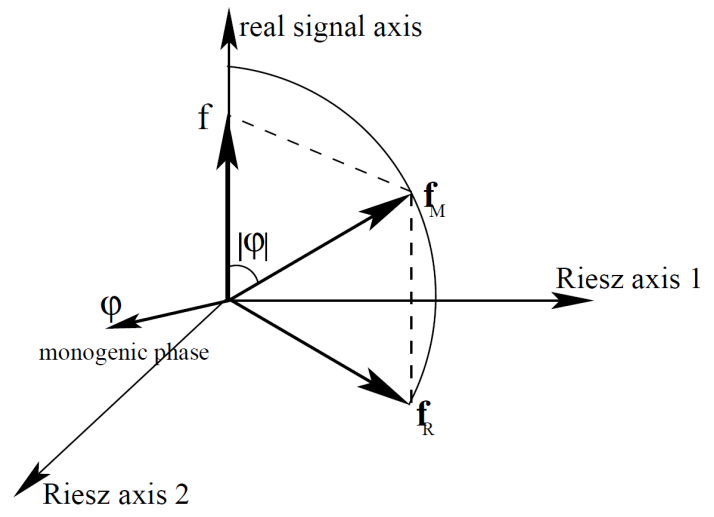

Fig. 1. Monogenic phase represented by means of a rotation vector. The amplitude of the rotation vector is the angle between the real signal and the monogenic signal. The rotation vector is orthogonal to the plane spanned by the real signal axis and $\mathbf{f}_{\mathcal{R}}$ [19].

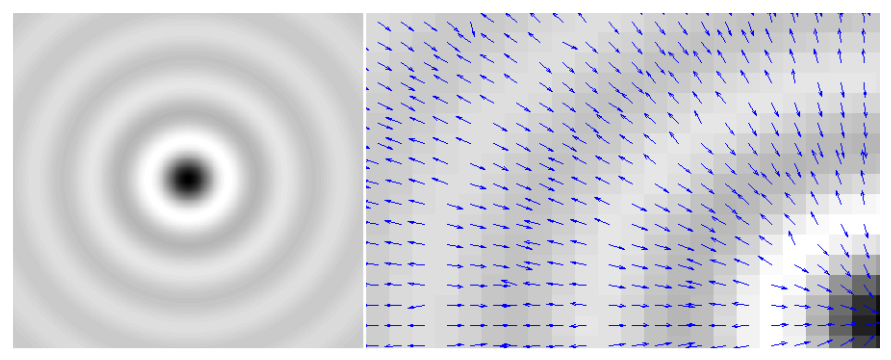

Fig. 2. 2D Sinus Cardinal (left). Monogenic local phase vector field for a subregion of the input signal with $\arctan (\cdot) \in[0, \pi]$ (right).

Several families of quadrature pairs have been proposed and applied in the literature. Most authors have not provided a reasonable justification for the use of a particular family apart from simplicity of use or the satisfaction of the zero DC condition. In [17], [18], Boukerroui, Noble and Brady compare several pairs of quadrature filters and concluded that log-Gabor kernels are probably not a very good choice in the case of feature detection. They showed that Cauchy family has better properties. In the frequency domain, 1D Cauchy kernel is defined by

$$
c(w)=w^{a} \exp (-s w), \quad a \geq 1,
$$

where $s$ is a scaling parameter and $a / s$ is the peak tuning frequency of the filter.

\section{The edge detection measure}

Step edge detection is performed using the feature asymmetry measure ( $F A$ ) of Kovesi [20] defined using the monogenic signal presented previously. Kovesi suggested to use $F A$ over a number of scales to detect step edge features. We define the multiple scales feature asymmetry $F A$ :

$$
F A=\sum_{s} \frac{\left\lfloor\left|\mathbf{o d d}_{s}\right|-\mid \text { even }_{s} \mid-T_{s}\right\rfloor}{\sqrt{\text { even }_{s}^{2}+\text { odd }_{s}^{2}}+\varepsilon},
$$

where $\lfloor\cdot\rfloor$ denotes zeroing of negative values and $T_{s}$ is the scale specific noise threshold [20]. The $F A$ takes values in 
$[0,1]$, close to zero in smooth regions and close to one near boundaries.

The application of this operator in [10], [8] for ultrasound images has yielded good results. The authors used $F A$ with steerable filter for boundary detection. However, it is expected to obtain better results using the monogenic signal, as it is the natural extension of the 1D analytical signal.

\section{LEVEL SET METHODS AND FRONT PROPAGATION}

In this section we present our proposed segmentation method. The idea is to use a novel phase based speed function within the level set framework.

Consider a gray level image as a function $I: \Omega \rightarrow \mathbb{R}^{+}$ where $\Omega \in \mathbb{R}^{2}$ is the image domain. The image gradient vector field is given by $\nabla I(x, y)$. Let us define the evolving contour $C:[0, L] \rightarrow \mathbb{R}^{2}$, given in a parametric form $C(s)=$ $\{x(s), y(s)\}$, where $s$ is an arclength parameter, and whose normal is defined by $\mathbf{n}(s)=\left\{-y_{s}(s), x_{s}(s)\right\}$.

The alignment term idea proposed by Kimmel and Bruckstein [21], [22], is to search for a contour $C$ that interacts with a given image, such that the curve's normal aligns with the gradient vector field. The alignment of the two vectors can be measured by their inner product that we denote by $\langle\mathbf{n}, \nabla I\rangle$. The geometric functional of the alignment measure in its robust form is given by [21], [22] :

$$
E_{A}(C)=-\int_{0}^{L}|\langle\mathbf{n}(s), \mathbf{V}(x(s), y(s))\rangle| d s,
$$

where $\mathbf{V}$ is a given vector field (for example gradient of image). The functional $E_{A}(C)$ measures the alignment between the local image orientations and the curve's normals. In the proposed work, we use the local orientation computed by means of the monogenic phase $\varphi$ given by (2) instead of the classical gradient estimation. As shown in Fig. 3, the monogenic phase offers a good orientation estimation of the edge. The inner product $\langle\cdot, \cdot\rangle$ gets high values if the curve's normal aligns with the local orientation of the image. This is useful in low contrast boundaries.

In our active contour model we will minimize the above alignment term and we will add some regularizing terms. It is known in literature that when we use several terms, geodesic active contour model (GAC) [23] serves as a good regularization for other dominate terms; like the alignment term in case we have good orientation estimation of the edge, see [22]. Therefore, we introduce the energy functional $E(C)$ defined by

$E(C)=-\int_{0}^{L}|\langle\mathbf{n}(s), \mathbf{V}(x(s), y(s))\rangle| d s+\lambda \int_{0}^{L} g(C(s)) d s$

The GAC term is an inverse edge indicator function, like $g(x, y)=1 /(1+|\nabla I|)$, along the contour. The search in this case, would be for a curve along which the inverse edge indicator gets the smallest possible values.

In our active contour model we also use a phase based geodesic active contour term. We define the phase based edge indicator function by:

$$
g=1-(F A)^{\alpha}
$$

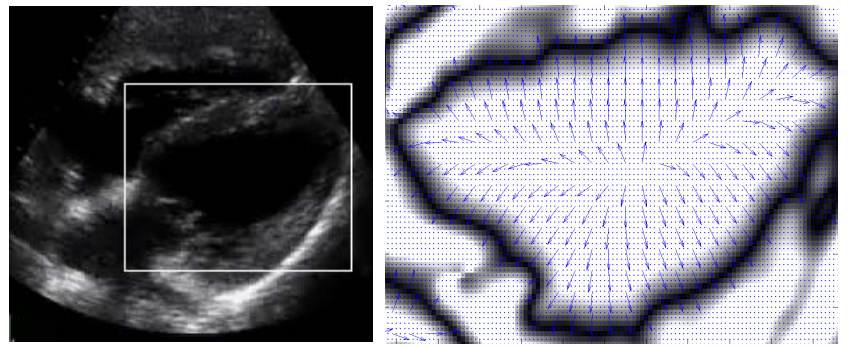

Fig. 3. Left: Original echocardiographic image of a four chamber view with a defined ROI. Right: the underlying grey value image shows the $g$ function and the vector field show the local phase vector field, both calculated on the showed ROI.

where $\alpha \in[0,1]$ is a hyperparameter and $F A \in[0,1]$ is the feature asymmetry measure, defined by (6). Thus the values of $g$ are close to one in smooth regions and close to zero near boundaries. As shown in Fig. 4, by moving closer to finer scales, the FA measure recovers details and discontinuities, but looses regularity and continuity of the boundaries.

Following [22], the gradient descent flow minimizing (8) to which we have added an area term as in [24], in the level set formulation, is given by:

$$
\begin{aligned}
\frac{\partial \phi}{\partial t}=[ & -\mu \operatorname{sign}(\langle\boldsymbol{\varphi}, \nabla \phi\rangle) \operatorname{div}(\boldsymbol{\varphi}) \\
& \left.+\lambda \operatorname{div}\left(g(x, y) \frac{\nabla \phi}{|\nabla \phi|}\right)+\nu g\right]|\nabla \phi|,
\end{aligned}
$$

where $\mu, \lambda$ and $\nu$ are a fixed parameters.

\section{PRELIMINARY RESUlts}

Matlab 7.6 (R2008a) was used for the implementation of the proposed method. In all the experimental results shown in this section, the following parameters were fixed as such : $s=15$, bandwidth $=1, \alpha=1, \nu= \pm 2$.

Fig. 4, shows the edge detection function applied on real ultrasound image. It is computed by the $F A$ measure, using the monogenic signal with the Cauchy filters family. By moving closer to the coarse scales, edge detection looses details but recovers regularity of the boundaries. This measure is injected in the speed function of a level set framework. Fig. 5 shows a comparison, on an echocardiographic image, of our proposed method against the well known GAC model. The left result obtained by our method shows more details and is more closely fit to the true boundaries than the GAC segmentation.

Finally, Fig. 6 shows illustrative results of our method on two typical ultrasound images. These preliminary results give the reader some insight regarding the robustness to speckle noise and to attenuation.

The proposed method gives accurate results in regions of low contrast, and seems to be robust against noise. The clinical experts are satisfied with these preliminary results. The specificities of ultrasound images respond well to local phase based processing, therefore we believe phase-based methods are well suited for the processing of ultrasound images. 
We aim to further develop the method in a multiple scales framework and generalize it for higher dimensions $(3 \mathrm{D}+\mathrm{t})$. It is also interesting to investigate the addition of region termes,

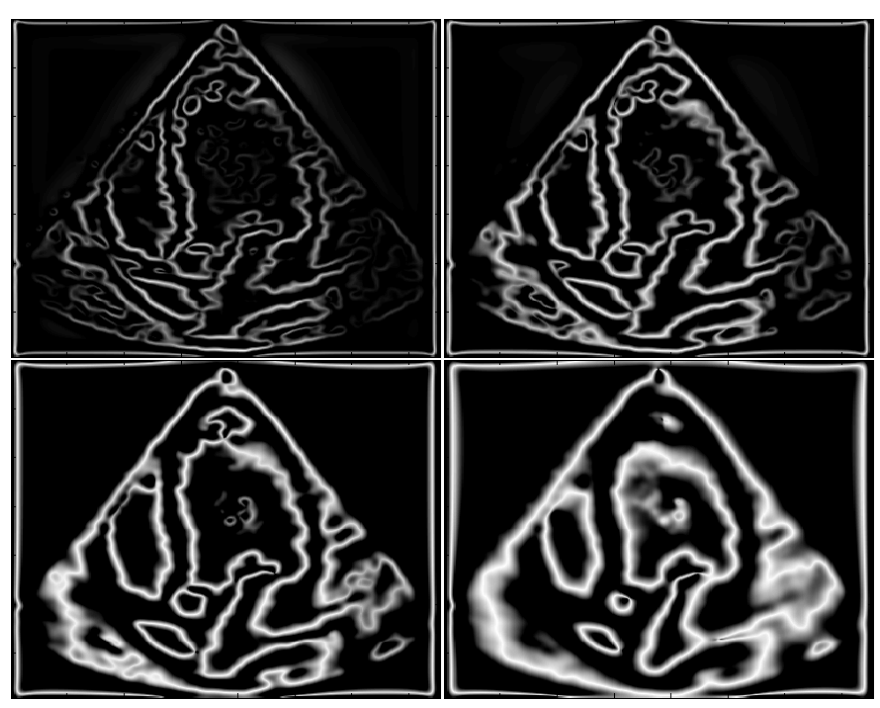

Fig. 4. Examples of feature asymmetry measure at different scales. From top-left to bottom-right: $s \in\{15,20,25,30\}$, bandwidth $=2.5$ ). The original image is given on Fig.6 top-left.

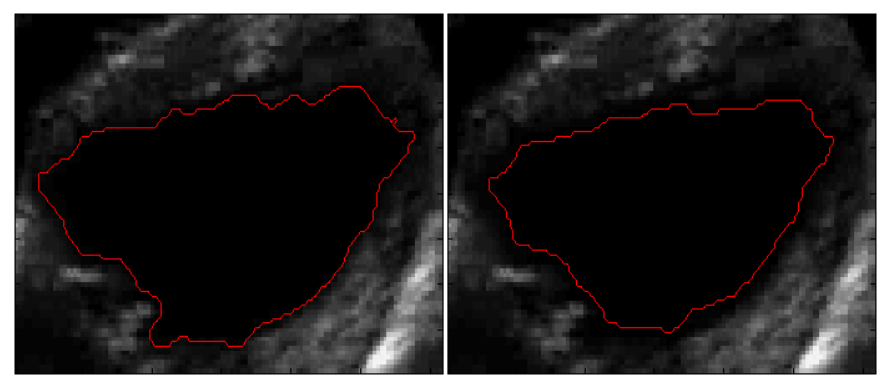

Fig. 5. Segmentation of the left ventricle. Left: result with our method, $\lambda=3, \mu=3.2$. Right: result of the GAC model, $\lambda=1.5, \mu=0 ; \sigma=2$.

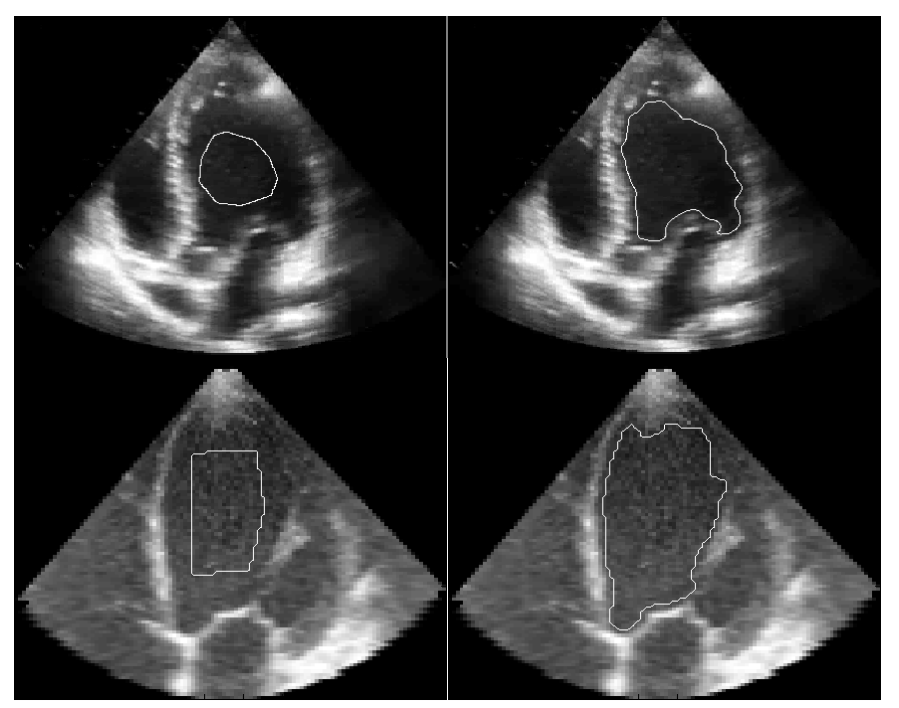

at least at the beginning of the segmentation process, in order to improve the capture range of the method.

\section{REFERENCES}

[1] J. A. Noble and D. Boukerroui, "Ultrasound image segmentation: A survey," IEEE Trans. Med. Imag., vol. 25, no. 8, pp. 987-1010, 2006.

[2] M. Felsberg and G. Sommer, "A new extension of linear signal processing for estimating local properties and detecting features," in In 22. DAGM Symposium Mustererkennung, 2000, pp. 195-202.

[3] B. Nr, M. Felsberg, and G. Sommer, "The monogenic signal," IEEE Trans. Signal Process., vol. 49, no. 49, pp. 3136-3144, 2001.

[4] M. Felsberg and G. Sommer, "The monogenic scale-space: A unifying approach to phase-based image processing in scale-space," J. Math. Imag. Vision, vol. 21, pp. 5-26, 2003.

[5] V. Grau, H. Becher, and J. Noble, "Registration of multiview real-time 3-D echocardiographic sequences." IEEE Trans. Med. Imag., vol. 26, no. 9, pp. 1154-65, 2007.

[6] J. Zhou, Y. Xu, and X. Yang, "Quaternion wavelet phase based stereo matching for uncalibrated images," Pattern Recogn. Lett., vol. 28, no. 12, pp. 1509-1522, 2007.

[7] A. Wong, "Fast communication: Adaptive bilateral filtering of image signals using local phase characteristics," Signal Process., vol. 88, no. 6, pp. 1615-1619, 2008.

[8] D. Boukerroui, J. A. Noble, and M. Brady, "Feature enhancement in low quality images with application to echocardiography," in IPMI. London, UK: Springer-Verlag, 2001, pp. 453-460.

[9] P. Kovesi, "Phase congruency detects corners and edges," in in The Australian Pattern Recognition Society Conference: DICTA 2003, 2003, pp. 309-318.

[10] M.-P. Miguel and N. J. Alison, " $2 \mathrm{D}+\mathrm{t}$ acoustic boundary detection in echocardiography," in MICCAI. London, UK: Springer-Verlag, 1998, pp. 806-813.

[11] I. Hacihaliloglu, R. Abugharbieh, A. Hodgson, and R. Rohling, "Bone segmentation and fracture detection in ultrasound using $3 \mathrm{~d}$ local phase features," in MICCAI. Berlin, Heidelberg: Springer-Verlag, 2008, pp. 287-295.

[12] G. Läthén, J. Jonasson, and M. Borga, "Phase based level set segmentation of blood vessels," in Proc. of 19th ICPR. Tampa, FL, USA: IAPR, December 2008.

[13] G. Läthén, J. Jonasson, and M. Borga, "Blood vessel segmentation using multi-scale quadrature filtering," Pattern Recognition Letters, vol. In Press, Accepted Manuscript, pp. -, 2009.

[14] R. Ali, M. J. Gooding, M. Christlieb, and J. M. Brady, "Phase-based segmenation of cells with brightfield microscopy," in IEEE Symposium on Biomedical Imaging, 2007, pp. 57-60.

[15] A. V. Oppenheim and J. S. Lim, "The importance of phase in signals," Proceedings of the IEEE, vol. 69, no. 5, pp. 529-541, May 1981.

[16] G. H. Granlund and H. Knutsson, Signal Processing for Computer Vision. Kluwer Academic Publishers, 1995.

[17] D. Boukerroui, J. A. Noble, and M. Brady, "On the choice of band-pass quadrature filters," J. Math. Imaging Vis., vol. 21, no. 1, pp. 53-80, 2004.

[18] — Frontiers in Robotics Research, Max A. Denket ed. Nova Publishers, 2006, ch. On the Selection of Band-Pass Quadrature Filters, pp. 67-111.

[19] R. San Jose Estepar, "Local structure tensor for multidimensional signalprocessing. applications to medical image analysis." Ph.D. dissertation, University of Valladolid, Spain, 2005.

[20] P. Kovesi, "Image features from phase congruency," Videre, Tech. Rep., 1999.

[21] R. Kimmel and A. M. Bruckstein, "Regularized laplacian zero crossings as optimal edge integrators," Int. J. Comp. Vision, vol. 53, pp. 225-243, 2003.

[22] V. Caselles, R. Kimmel, and G. Sapiro, Handbook Of Image And Video Processing, A.C. Bovik 2nd revised ed. Academic Press Inc, 2005, ch. Geometric active contours for image segmentation, pp. 613-629.

[23] _ , "Geodesic active contours," Int. J. Comp. Vision, vol. 22, no. 1, pp. 61-79, 1997.

[24] C. Li, C. Xu, C. Gui, and M. D. Fox, "Level set evolution without reinitialization: A new variational formulation," in IEEE CVPR, vol. 1, San Diego, 2005, pp. 430-436.
Fig. 6. Left: initial contours. Right: phase based segmentation results of the left ventricle, $\mu=1, \lambda=1$. 